\title{
Triboelectric Properties of a Ferroelectric Materials and Its Application in Energy Harvesting
}

\author{
Amir Shahzad, K. Rohana Wijewardhana, E. N. Jayaweera, Jang-Kun Song \\ School of Electronics \& Electrical Engineering, Sungkyunkwan University \\ Jangan-gu Suwon Gyeonggi-do, 16419, South Korea \\ shahzad@skku.edu; rohanakg@skku.edu; eranjiya@gmail.com; jk.song@skku.edu
}

\begin{abstract}
Contact electrification, from untapped sources in our environment has been suggested as an alternative way for electricity generation with the global energy crisis as compared to burning fossil fuels. Tribo effect with electrostatic induction is vastly used for contact electrification for energy generation. Usually, a material has same triboelectric properties along the surface, it can either generate positive charge or negative tribo charge after come in contact, depending on the relative position in triboelectric series. But, ferroelectric materials has a unique property that its triboelectric properties can be controlled via its poling direction and magnitude. Generally, in highly poled layer, one side has completely opposite triboelectric properties as compared to the other side. That is, if positive charge will appear on one side than negative charge on other side after come in contact with same metallic material. This communication will briefly discuss about the triboelectric properties of a poled ferroelectric material. It also introduces a different configured triboelectric generator consisting of one polymer layer that generates electricity when both sides of the layer come in contact with same metallic electrode.
\end{abstract}

Keywords: Triboelectrification, Ferroelectric Material, Negative and Positive Poled Surfaces.

\section{Introduction}

Micro-energy harvesting for sensors and small electronic devices, and other feasible applications have been attracted an increasing attention by using the contact electrification[1], piezoelectric effect[2], electric double layer modulation.[3] These different methods are associated with scavenging energy from mechanical vibrations, human motion, air bubble or water droplets, and wind. Particularly, in contact electrification when two different materials come in contact with each other bear charges which are opposite in signs. Usually a material can bear only one kind of charge depending on its relative position in triboelectric series. It was found that triboelectric properties of a ferroelectric material could be controlled with the varying electrical poling of that material, therefore its place on the triboelectric series changes in accordance with the poling direction and magnitude. Recently, by using this property of ferroelectric material, a triboelectric generator using human skin was shown which left almost zero charge on the skin.[1] Therefore, surface potential of any material could become smaller or larger after rubbing with the poled ferroelectric polymer and this depends on poling direction. Electrical poling of a ferroelectric material is done by providing a high electric field across the layer. During the application of high electric field, the positive poles of the material settles down along one side of the material while negative poles settles along the opposite side. Therefore, a poled layer of ferroelectric material could bear both kind of charges when it comes in contact with same material, the polarity of tribo charges depend on the side of the layer.

\section{Experimentation and working}

For experiments, we prepared a solution of 20(wt. \%) poly (vinylidenefluoride-co-trifluoroethylene) PVDF-TrFE copolymer, a ferroelectric material, in N, N-dimethylformamide (DMF) solution and made a thin layer on a flexible substrate. After curing the layer, it was peeled off from the flexible substrate. Then the layer was polarize by applying high electric field as shown in fig. 1(a). The surface on one side of layer was positively poled (red region in image) while the other was negatively poled (blue region) as shown in right image of fig. 1(a). Aluminium has triboelectric properties in between the positive poled and negative poled PVDF-TrFE surface. By tapping the two aluminium electrodes from both sides of 
PVDF-TrFE TEG, an AC voltage was generated. The output current was measured with a $1.2 \mathrm{M} \Omega$ load resistor using a digital multimeter (DMM 7510, Keithley, USA). The output voltage and current signals are shown in fig.1(c).

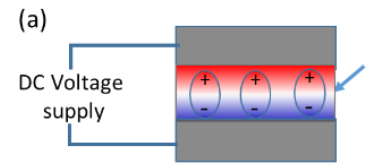

(b)

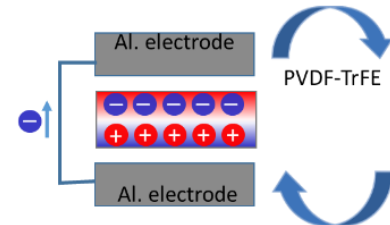

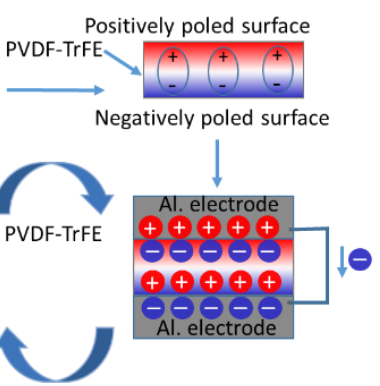

(c)

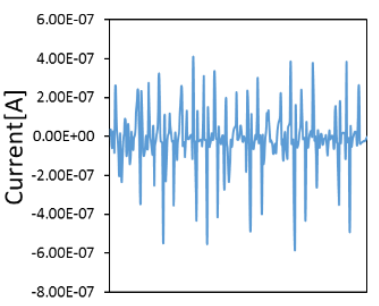

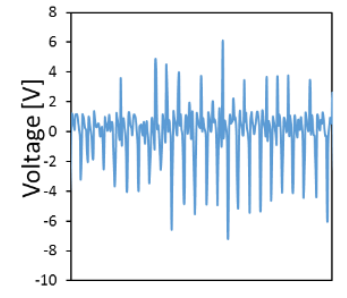

Fig. 1: (a) Schematic diagram for poling PVDF-TrFE polymer red shaded region of polymer layer is positively poled while blue shaded region is negatively poled. (b) Mechanism of triboelectric generator (encircled charges are tribocharges). (c) Output current and voltage signals of the TEG.

The working mechanism of proposed TEG is schematically shown in Fig. 1(b). The TEG device is composed of a single material. One side of material has a higher electron affinity that is ready to release electron when come in contact with some other material while other side of same material has opposite property of that. Therefore, when aluminium electrode comes in contact with the PVDF-TrFE layer from both sides, tribocharges appears on the interfaces depending on the relative triboelectric properties of the contacting materials. The positive and negative poled sides of polymer got negative and positive tribocharges respectively after come in contact with aluminium electrode. These tribocharges are confined to the surface and stay on the surface for an extended period even after separation; moreover it is difficult to move these charges. Now, when aluminium electrode come again in contact with both sides simultaneously, the electric equilibrium will disturb due to the different tribocharges on upper and bottom surfaces of the ferroelectric material. The bottom side is at higher potential to that of top side as in fig 1 (b), therefore an electrostatic induction of charges take place inside the aluminium electrodes and current will flow from higher potential towards lower potential and when metallic electrodes separate from the material then current will flow in opposite direction and this cycle continues.

\section{Conclusion}

In summary, this study shows that polarized ferroelectric material can change its triboelectric properties with its poling direction. Therefore, when it come into contact with metallic electrode it either supply or withdraw negative charges to/from electrode depending on the poling direction of the material. Positive poled surface tends to withdraw electron after aluminium/PVDF-TrFE contact therefore negative charge appears while negative poled surface will get positive tribocharges. An electrically poled ferroelectric layer is positively poled in one side while other side is negatively poled. By using this layer, a simple TEG was also shown when similar kind of metallic material come in contact from both sides of the ferroelectric material. This layer can be used to control the potential of any surface after rubbing with it, by adjusting the poling direction and magnitude of the ferroelectric material.

\section{Acknowledgements}

This work was supported by the National Research Foundation of Korea (NRF) funded by the ministry of Science and ICT (NRF- 2014R1A2A1A11054392).

\section{References}

[1] A. Shahzad, K. R. Wijewardhana, and J.-K. Song, "Comment on 'An ultrathin stretchable triboelectric nanogenerator with coplanar electrode for energy harvesting and gesture sensing' by X. Chen, Y. Song, H. Chen, J. Zhang and H. Zhang," Journal of Materials Chemistry A, 2017, 5 , 12361," J. Mater. Chem. A, vol. 0, pp. 1-3, 2017.

[2] S. Siddiqui, D. Il Kim, L. T. Duy, M. T. Nguyen, S. Muhammad, W. S. Yoon, and N. E. Lee, "High-performance flexible lead-free nanocomposite piezoelectric nanogenerator for biomechanical energy harvesting and storage," 
Nano Energy, vol. 15, pp. 177-185, 2015.

[3] K. R. Wijewardhana, T. Z. Shen, and J. K. Song, "Energy harvesting using air bubbles on hydrophobic surfaces containing embedded charges," Appl. Energy, vol. 206, no. April, pp. 432-438, 2017. 